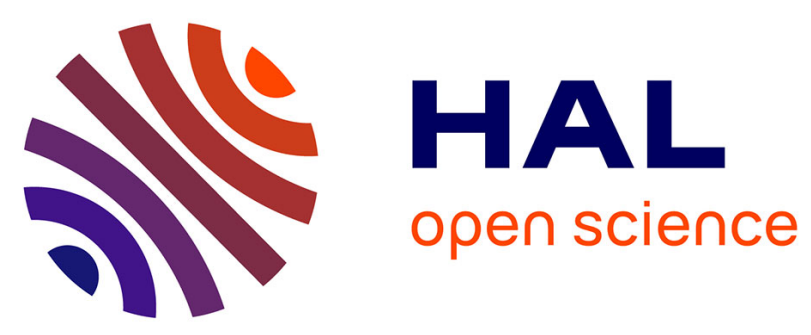

\title{
Sensitivity and Noise Evaluation of a Bonded Magneto(elasto)Electric Laminated Sensor Based on In-plane Magneto-capacitance Effect for Quasi-static Magnetic Field Sensing
}

Xin Zhuang, Marc Lam Chok Sing, Christophe Dolabdjian, Yanping Wang, Peter Finkel, Jiefang Li, Dwight Viehland

\section{To cite this version:}

Xin Zhuang, Marc Lam Chok Sing, Christophe Dolabdjian, Yanping Wang, Peter Finkel, et al.. Sensitivity and Noise Evaluation of a Bonded Magneto(elasto)Electric Laminated Sensor Based on In-plane Magneto-capacitance Effect for Quasi-static Magnetic Field Sensing. IEEE Transactions on Magnetics, 2015, pp.10.1109/TMAG.2014.2356852. 10.1109/TMAG.2014.2356852 . hal-01162288

\author{
HAL Id: hal-01162288 \\ https://hal.science/hal-01162288
}

Submitted on 10 Jun 2015

HAL is a multi-disciplinary open access archive for the deposit and dissemination of scientific research documents, whether they are published or not. The documents may come from teaching and research institutions in France or abroad, or from public or private research centers.
L'archive ouverte pluridisciplinaire HAL, est destinée au dépôt et à la diffusion de documents scientifiques de niveau recherche, publiés ou non, émanant des établissements d'enseignement et de recherche français ou étrangers, des laboratoires publics ou privés. 


\title{
Sensitivity and Noise Evaluation of a Bonded Magneto(elasto)Electric Laminated Sensor Based on In-plane Magneto-capacitance Effect for Quasi-static Magnetic Field Sensing
}

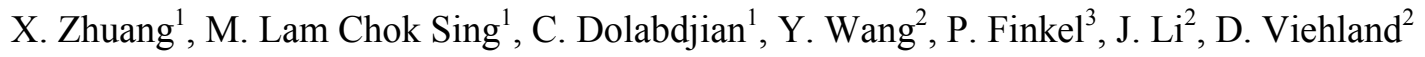 \\ ${ }^{1}$ Normandie Univ, France; UCBN, GREYC, F-14032 Caen, France; CNRS, UMR 6072, F-14032 Caen, France \\ ${ }^{2}$ Materials Science and Engineering, Virginia Tech., Blacksburg, VA 24061, USA \\ ${ }^{3}$ U.S. Naval Research Laboratory, Washington, D.C. 20375
}

\begin{abstract}
The quasi-static magnetic field detection of a layer-bonded Magneto(elasto)Electric (ME) laminate has been investigated by measuring the in-plane electric capacitance via its interdigital electrodes close to the piezoelectric resonant frequency. The ME layered composite is considered as a stress-induced dielectric effect, because there is practically no direct response of the electric capacitance to an external magnetic field [1]. The sensitivity is dominated by the magneto-elastic coupling in the magnetic layer and on the stress which is induced by the permittivity change in the piezoelectric layer. The low frequency magneto-capacitance effect is sensitive to an external magnetic bias which can modulate the electric permittivity by producing a stress. The magnetoelastic coupling is another important parameter for this magnetic field detection mode. For a given magnetic field, the amplitude of the magnetostriction is directly related to this parameter, too [2]. Therefore, an optimal magnetic bias can maximize the induced strain or stress which is coupled into the piezoelectric layer through the change of the electric permittivity in this layer. To evaluate the sensitivity and the noise performance based on the magneto-capacitance effect, we have used the piezoelectric and magnetic constitutive equations to predict the permittivity dependence [16]. Experimentally, this sensor achieved an equivalent magnetic noise spectral density, presently still limited, by the noise of the detection electronics, $\sim 100 \mathrm{pT} / \sqrt{ } \mathrm{Hz}$ at $1 \mathrm{~Hz}$ and offered a DC detection capability. Based on the model and experimental nonlinear factors, an equivalent sensor noise spectral density close to the $\mathrm{pT} / \sqrt{ } \mathrm{Hz}$ can be ultimately predicted taking into account the mechanical loss limitation of the sensor [16].
\end{abstract}

Index Terms - magnetoelectric, magnetic noise, modulation

\section{INTRODUCTION}

A layered magnetoelectric (ME) laminated sensor consists of a magnetostrictive (MS) phase, a piezoelectric (PE) phase, bonding layers and associated electrodes. A magnetic induced strain can be generated in the MS phase via the magnetostrictive effect. This strain is coupled into the piezoelectric layer via the bonding layer. Many enhancements have been carried out by choosing the material and adjusting the ratio between the MS and PE phases of the composite [3], [4], [5], [6]. Thereafter, noise sources in the laminate have been investigated to evaluate the expected noise performance for magnetic sensor applications. For years, both intrinsic noise sources in the composite and extrinsic noise sources in the first amplification stage have been analyzed [7], [8], [9], [10], [11]. The ultimate noise performance of a ME layered laminate has been mathematically modeled and experimentally verified by means of diverse modeling and noise spectrum measurements [11]. It is mainly limited by the dielectric loss noise in the piezoelectric layers which result from electric dissipations induced by the electric domain wall random motions [12], [13]. Under an electric field excitation, especially at the piezoelectric resonance, the ME laminate can

Manuscript received Xx, 2014. Corresponding author: X. Zhuang (email: xin.zhuang@unicaen.fr).

Digital Object Identifier inserted by IEEE serve as a magneto-capacitance magnetic field sensor. This effect is based on the parametric modulation of the electric permittivity by a low-frequency magnetic-field-induced stress [14]. The magnetic field sensing capacitance of a ME laminate reaches it maximum around the piezoelectric resonant frequency, where the efficient dielectric coefficient is most sensitive to a low frequency external stress [15]. This detection method depends on the amplitude modulation of an excitation carrier which is actively driven by a sinusoidal electric field at a relatively high frequency. The low-frequency signal can be recovered from the envelope of the carrier by using a classical amplitude demodulation process.

\section{SENSITIVITY AND NOISE FLOOR}

\section{1) Sensor description and experimental setup}

A ME laminate consists of three coupled layers. A PE layer consisting of 5 macro-fibers $\left(40 \times 2 \times 0.2 \mathrm{~mm}^{3}\right)$ is sandwiched between two MS layers. Each MS layer is made of three bidimensional Metglas sheets $\left(80 \times 10 \times 0.15 \mathrm{~mm}^{3}\right)$. The resulting ME laminate has a geometry of $80 \times 10 \times 0.35 \mathrm{~mm}^{3}$. Two pairs of interdigital electrodes are disposed on both top and bottom surface of the PE layer with a center-to-center spacing of $850 \mu \mathrm{m}$. Thus, the electric signal can be sensed from the piezoelectric layer. Electrodes are connected to the negative input of a charge amplifier as shown in the Fig. 1. A sinusoidal excitation is applied on the positive input of the amplifier so as to electrically excite the ME laminated (cf. figure 1). 


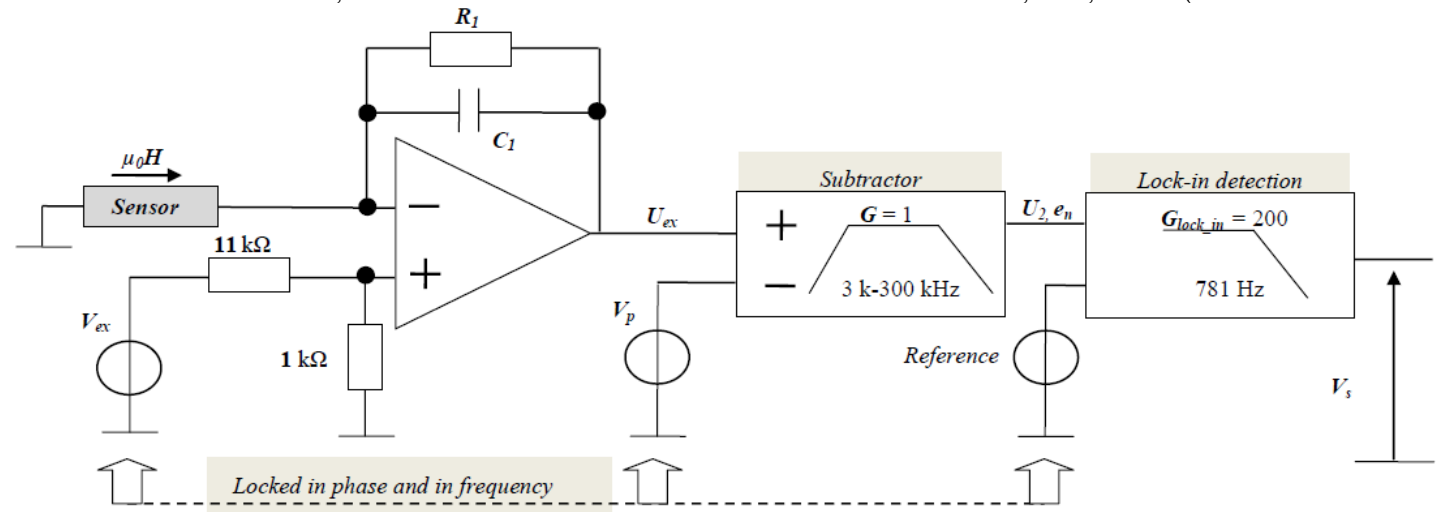

Fig. 1. Experimental scheme of the magneto-capacitance modulation and detection. The three locked generator used are HP33120A defined as [16]

The output signal from the charge amplifier is applied to one input of a subtracting amplifier having a $[3 \mathrm{kHz}, 300 \mathrm{kHz}]$ bandwidth. A compensation of the carrier signal is made via the second input, in order to reduce its amplitude before the demodulation process with the lock-in amplifier [17]. The applied low frequency magnetic field signal is recovered by using the excitation signal as the reference signal.

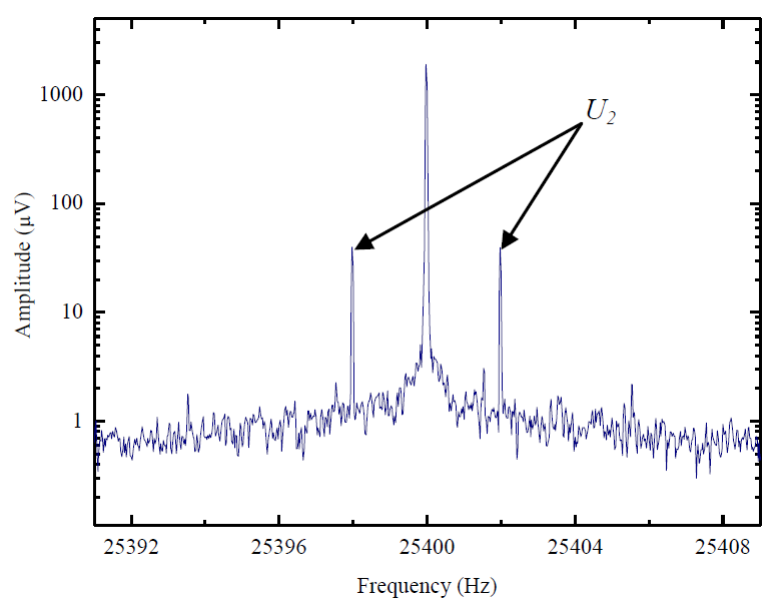

Fig. 2. Details of sideband reference signal (@2 Hz) around the excitation frequency appearing at the output $U_{2}$.

\section{2) Nonlinearity and sensitivity}

A sinusoidal magnetic field has been applied on the $\mathrm{ME}$ laminate along the longitudinal direction so that a low frequency reference signal $(@ 2 \mathrm{~Hz})$ is achieved. Here, the study is made for one freauency. However, the mqgnetic sensing content a bandzidth from DC to several hundred $\mathrm{Hz}$ according to the measurement. Fig. 2 represents the power spectrum curve of the output signal at the differential amplifier, showing the near carrier reference signal around the excitation carrier frequency of $25.4 \mathrm{kHz}$. These two peaks correspond to the frequency shifting of the low frequency reference signal around the excitation carrier frequency via the non-linearity in the ME laminate, the latter acting as a modulator at $25,400 \pm 2 \mathrm{~Hz}$ with an amplitude of $G \times U_{2}$ for each peak. $G$ is the gain of the subtractor. The nonlinear sensitivity is defined by the ratio between the carrier amplitude and that of one of the sideband reference signal at the output of the charge amplifier. The amplitude and noise spectrum curves were taken before the demodulation process in order to investigate the nonlinearity factor which can be

$$
\gamma_{1}=\frac{U_{2}}{U_{e x} H} .
$$

where $U_{e x}, H$ are the amplitude of the carrier appearing at the output of the charge amplifier and the amplitude of the external applied magnetic field as low frequency reference signal, respectively.

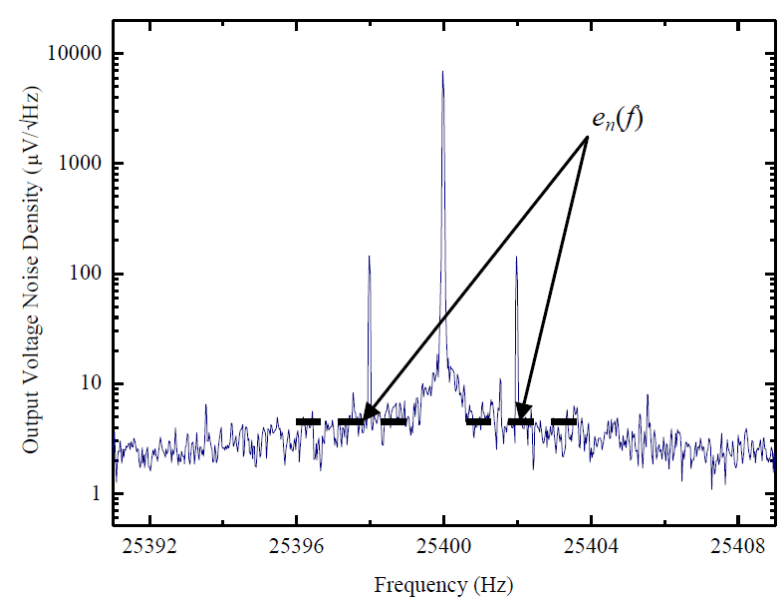

Fig. 3. Noise floor evaluation around the excitation frequency appearing at the output $U_{2}$.

The noise floor around the excitation frequency is given in Fig. 3. After the demodulation process, this noise level is transferred back to low-frequencies by considering the reference excitation signal. The ratio between the noise floor and the two peak amplitudes $U_{2}$ in Fig. 2 determines the noise performance for the magneto-capacitance modulation.

\section{3) Noise evaluation}

The expected intrinsic noise floor depends on the flexibility fluctuations due to mechanical dissipation. However, the observed noise level from the experiment was several orders higher than the expected theoretical noise level. This is due to a low nonlinear coefficient value and to the noise level induced by the sine wave generator used for the excitation signal and to the detection electronics. Firstly, the near carrier noise occurred because of the instability of the generator close to the carrier frequency as amplitude or phase noise, despite its high quality (close to the state of the art performance). This induces a $1 / f$ noise contribution which passes into the demodulation process. Secondly, the equivalent input voltage noise floor of the charge amplifier dominates the white noise 
floor.

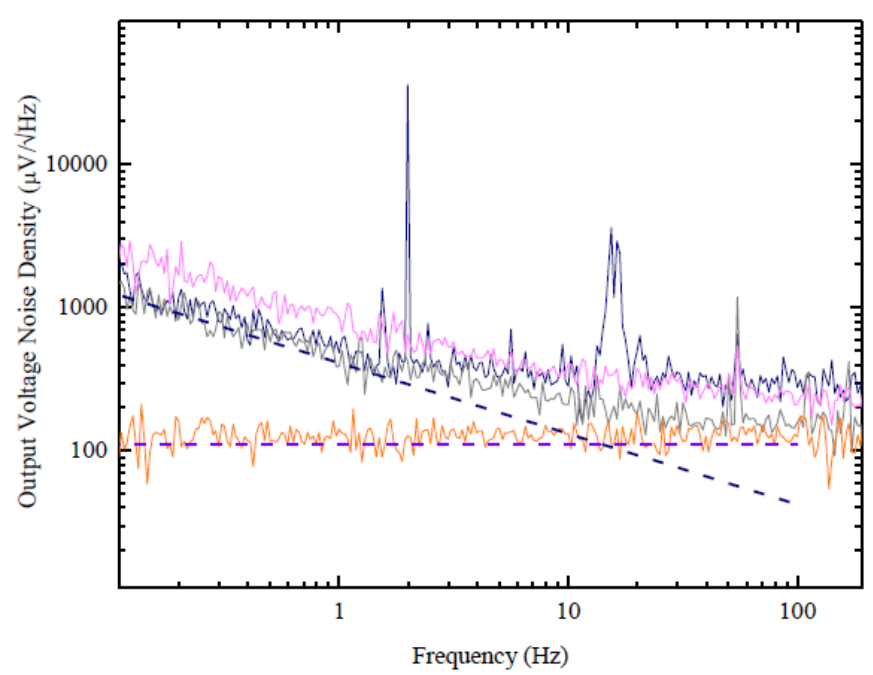

Fig. 4. Output voltage noise spectral density, $V_{s}$, as a function of the frequency.

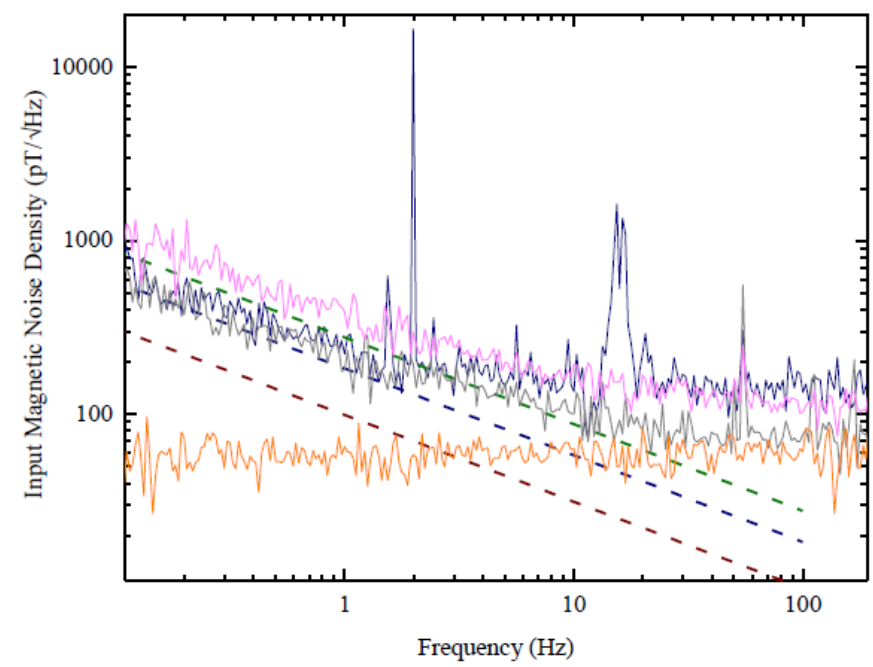

Fig. 5. Equivalent magnetic noise floor of the sensor as a function of the frequency.

Besides, the current noise of the resistance in the feedback loop of the charge amplifier produces a noise contribution as well. Taking the two noise sources, the output voltage noise expression under a certain voltage excitation is

$$
\begin{aligned}
e_{n}^{2}\left(f_{e x} \pm f\right) & =\left(\frac{1}{2 \pi f_{e x} C_{1}}\right)^{2}\left[i_{n_{-} R_{1}}^{2}\left(f_{e x} \pm f\right)+i_{n_{-} \text {amp }}^{2}\left(f_{\text {ex }} \pm f\right)\right] \\
& +\left(\frac{C+C_{1}}{C_{1}}\right)^{2}\left[e_{n_{-} \text {amp }}^{2}\left(f_{\text {ex }} \pm f\right)+e_{n_{-} \text {exc }}^{2}\left(f_{\text {ex }} \pm f\right)\right]
\end{aligned}
$$

where $i_{n_{-} R 1}\left(f_{\text {exc }} \pm f\right)$ is the current noise from the resistance $R_{l}, i_{n_{-} a m p}\left(f_{\text {exc }} \pm f\right)$ and $e_{n_{-} a m p}\left(f_{\text {exc }} \pm f\right)$ are the equivalent input current and voltage noise sources of the operational amplifier, respectively, $e_{n_{-} e x c}\left(f_{\text {exc }} \pm f\right)$ is the noise of the sine wave excitation generator, $C$ and $C_{I}$ are the sensor capacitor and the feed-back capacitor, respectively. By means of a classical demodulation process, the transfer function is observed with a value of $2.2 \times 10^{6}$ (in V/T). The output voltage noise floor and the equivalent magnetic noise floor are given in Fig. 4 and Fig. 5.

\section{NOISE PERFORMANCE CHARACTERIZATION}

The noise performance characterization of a ME laminate consists of 3 parts: Transfer function, output voltage noise and the equivalent magnetic noise characterization.

\section{1) Magnetic signal transfer function}

This sinusoidal magnetic field reference is fixed as a sweeping frequency. The ratio between the output of the lockin amplifier and this signal permit evaluation of the transfer function. Considering the constitutive equation, we calculated the sensitivity in magneto-capacitance modulation mode [16]

$$
\alpha_{M E_{-} E E}=\gamma_{1} \alpha_{E E} E_{e x c}
$$

where, $\alpha_{E E}$ is the direct electronic voltage sensitivity which is defined by

$$
\alpha_{E E}=\frac{\partial Q}{\partial E}=\varepsilon_{33, p} A_{p}+\varphi_{p}^{2} C_{m e c h} .
$$

where $E$ and $Q$ are respectively the applied voltage field and generated charge value across the interdigital electrodes. $\varepsilon_{33, p}$ and $A_{p}$ are respectively the in-plan permittivity and the section surface of the piezoelectric layer. $\varphi_{\mathrm{p}}$ is the piezoelastic coupling coefficient and $C_{\text {mech }}$ is the total mechanical capacitance of the composite. This equation consists of a nonelastic term and an elastic term [16]. The magneto-capacitance modulation mechanism is due to the piezoelastic coupling, $\varphi_{\mathrm{p}}$, change in function of a magnetic-induced stress. Here, the stress was produced by the low frequency magnetic field. So, by applying this reference signal the demodulated output was maximized at low frequency. The transfer function was measured by using a frequency-sweeping sinusoidal signal in the working bandwidth of the system under the modulation mode. It yields

$$
T r_{M E_{-} E E}=\frac{\alpha_{M E_{-} E E}}{C_{1}} \times G \times G_{\text {lock_in }}
$$

where $G_{\text {lock } \_ \text {in }}$ is the gain of the lock-in amplifier.

\section{2) Equivalent magnetic noise floor}

The equivalent magnetic noise of the ME composite under the magneto-capacitance working mode was characterized by the ratio between the output voltage noise spectral density and the magnetic transfer function. In Fig. 4, the dashed blue line and the dashed purple are respectively the simulation for the noise limits induced by the generator and by the voltage noise source of the charge amplifier. Gray and orange curves are respectively the measured limitation from the generator and lock-in amplifier. Above these curves, the noise level in blue and in pink are respectively the output voltage noise level for the magneto-capacitance modulation techniques with the sensor and the one with a passive capacitor (which value is 
close to the sensor capacitance) for the present setup.

Shown in Fig. 5 is the equivalent magnetic noise of the detection electronics. The noise measurement were made in a magnetic shielding room to avoid the environmental magnetic noise influence. The curves in the Fig. 5 are the equivalent noise levels referring to the output voltage noise limitation and the low frequency magnetic field transfer function of the magneto-capacitance modulation with present nonlinear coefficient. The colors correspond to the ones in Fig. 4 with two additional dashed lines in dark green and dark red for the top and bottom limitations. Several $100 \mathrm{pT} / \sqrt{\mathrm{Hz}}$ was achieved at $1 \mathrm{~Hz}$ and $100-150 \mathrm{pT} \sqrt{ } \mathrm{Hz}$ was achieved at white bandwidth. These limitations are due to the incertitude of the noise limitation from the generator. So, we found that the excitation noise from the generator is the main contribution at low frequency. However, the amplifier voltage noise gives the main noise contribution in the white regime.

\section{CONCLUSION}

By using the magneto-capacitance effect, a quasi-static magnetic field can be sensed in a layered ME composite by measuring the modulated electric capacitance. This effect was used for low frequency magnetic field sensing. The output sensitivity depends on the nonlinearity in the ME laminate. Noise limitations have been analyzed and experimentally measured by considering the noise contribution from the excitation and detection electronics. Based on the magnetocapacitance effect, the equivalent magnetic noise floor was still limited by the instability from the excitation electronics in low frequency ( $1 / f$ noise) and from the detection electronics in white frequencies, presently. Besides, a further higher performance is expected by reducing the electronic noise from the excitation and detection circuits and by improving the nonlinear factor, $\gamma_{1}$, of the laminate.

\section{ACKNOWLEDGMENT}

The authors gratefully acknowledge the full support by the O.N.R. (Office of Naval Research) Global.

\section{REFERENCES}

[1] N. A. Pertsev, S. Prokorenko, and B. Dkhil, "Giant magnetoelectric of strained ferroelectric-ferromagnetic hybrids," Physical Review B, vol. 85 , p. 134111,2012

[2] D. C. Jiles, "Theory of the magnetomechanical effect," Journal Physics D: Applied Physics, vol. 28, pp. 1537-1546, 1995

[3] G. Sreenivasulu, U. Laletin, V. M. Petrov, V. V. Petrov, and G. Srinivasan, "A permendur-piezoelectric multiferroic composite for lownoise ultrasensitive magnetic field sensors," Applied Physics Letters, vol. 100, p. 173506, 2012

[4] S. Dong, J. Cheng, J. F. Li, and D. Viehland, "Enhanced magnetoelectric effects in laminate composites of Terfenol- $\mathrm{D} / \mathrm{Pb}(\mathrm{Zr}, \mathrm{Ti}) \mathrm{O}_{3}$ under resonant drive," Applied Physics Letters, vol. 83, p. 4812, 2003

[5] Z. Fang, S. G. Lu, F. Li, S. Datta, Q. M. Zhang, and M. EI Tahchi, "Enhancing the magnetoelectric response of Metglas/polyvinylidene fluoride laminates by exploiting the flux concentration effect," Applied Physics Letters, vol. 95, p. 112903, 2009

[6] H. Greve, E. Woltermann, H. -J. Quenzer, B. Wagner, and E. Quandt, "Giant magnetoelectric coefficients in $\left(\mathrm{Fe}_{90} \mathrm{Co}_{10}\right)_{78} \mathrm{Si}_{12} \mathrm{~B}_{10}$-AIN thin film composites," Applied Physics Letters, vol. 96, p. 182501, 2010
[7] Y. Wang, D. Gray, D. Berry, J. Gao, M. Li, J. Li, and D. Viehland, "An extremely low equivalent magnetic noise magnetoelectric sensor," Advanced Materials, vol. 23, pp. 4111-4114, 2011

[8] X. Zhuang, M. Lam Chok Sing, C. Cordier, S. Saez, C. Dolabdjian, J. Das, J. Gao, J. Li, and D. Viehland, "Analysis of noise in magnetoelectric thin-layer composites used as magnetic sensors," IEEE Sensors Journal, vol. 11, p. 2183, 2011

[9] S. Marauska, R. Jahns, H. Greve, E. Quandt, R. Knochel, and B. Wagner, "MEMS magnetic field sensor based on magnetoelectric composites," Journal of Micromechanics and Microengineering, vol. 22, p. 065024,2012

[10] Z. Xing, J. Li, and D. Viehland, "Modeling and the signal-to-noise ratio research of magnetoelectric sensors at low frequency," Applied Physics Letters, vol. 91, p. 142905, 2007

[11] X. Zhuang, C. Cordier, S. Saez, M. Lam Chok Sing, C. Dolabdjian, J. Gao, J. F. Li, and D. Viehland, "Theoretical analysis of the intrinsic magnetic noise spectral density of magnetostrictive-piezoelectric laminated composites," Journal of Applied Physics, vol. 109, p. 124512, 2011

[12] Y. Zhuang, S. O. Ural, A. Rajapurkar, S. Tuncdemir, A. Amin, and K. Uchino, "Derivation of piezoelectric losses from admittance spectra," Japanese Journal of Applied Physics, vol. 48, p. 041401, 2009

[13] F. A. Levinzon, "Noise of piezoelectric accelerometer with integral FET amplifier," IEEE Sensors Journal, vol. 5, p. 1235, 2005

[14] Q. Liu, X. Bian, J. Zhou, and P. Liu, "Colossal magnetodielectric effect cause by magnetoelectric effect under low magnetic field," Indian Academy of Sciences, vol. 34, pp. 238-286, 2011

[15] C. Israel, V. M. Petrov, G. Srinivasan, and N. D. Mathur, "Magnetically tuned mechanical resonances in magnetoelectric multilayer capacitors," Applied Physics Letters, vol. 95, p. 072505, 2009

[16] X. Zhuang, M. Lam Chok Sing, C. Dolabdjian, Y. Wang, P. Finkel, J. $\mathrm{Li}$, and D. Viehland, "Mechanical noise limit of a strain coupled magneto(elasto)electric sensor operating under a magnetic or an electric field modulation around its resonant frequency," IEEE Sensors, to be submitted

[17] M. Malatek, B. Dufay, S. Saez, C. Dolabdjian, "Improvement of the offdiagonal magnetoimpedance sensor noise," Sensors \& Actuators A, vol. 204 , pp. $20-24,2013$

Xin Zhuang was born in Qingdao, China, in 1983. He received the first B.S. degree in electronic science and technology from the Ocean University of China, Qingdao, and a second B.S. degree in electronics electrical engineering and automation and the M.S. degree in signal and circuits from the University of Brest, Brest, France, in 2007 and 2009, respectively. Since 2009 he has been with the Department of Electronics at GREYC Laboratory, ENSICAEN and the University of Caen Lower Normandy, Caen, France, where he received his $\mathrm{Ph} . \mathrm{D}$. degree in electronics and instrumentation. He is currently working as a post-doc fellow at GREYC Laboratory for performance optimization of magnetic sensors. His main research focus is on the noise performance optimization of magnetoelectric laminated composites.

Marc Lam Chok Sing received the Engineer degree from the Ecole Nationale Supérieure d'Ingénieurs de Caen (ENSICAEN), France, in 1985 and the $\mathrm{Ph}$.D. degree in Science from the University of Caen, Caen, France, in 1989. He is currently a Lecturer in Electronics at the ENSICAEN School of Engineering. His present research interests include magnetic sensors, highresolution magnetometers and low-noise electronics.

Christophe Dolabdjian was born in Enghien-les-Bains, France, in 1967. He received the M.S. and the Ph.D. degrees in electronics and instrumentation from the University of Caen, Caen, France, in 1991 and 1994, respectively, and the Habilitation Diploma in 2000.

In 1994, he joined the Groupe de Recherche en Informatique, Image, automatique et Instrumentation de CAEN CNRS UMR 6072 of ENSICAEN and the University of CAEN as an Assistant Professor, where he has been a Professor of Electronics, since 2001. His research interests included studied, development, optimization, improvement and comparison of numerous very high sensitivity and very low magnetic noise sensors (SQUID, JFM, Flux-gate, GMR, GMI, $\mu$ Hall, ME, Hybrid...), as well as their integration in applications, in open or close environment, like Biomagnetism and Non-Destructive Testing. He was also an Assistant Director and Director of the Doctoral School "SIMEM" from 2002 to 2006 and from 2007 to 2009 , respectively. Presently, he is the "Head of the Electronic Team" of the 
GREYC since 2007 and in charge of the "Licence Pro MCA" professional B.A. of the UCBN since 2001 .

Yaojin Wang is a Postdoctoral Associate of the Materials Science and Engineering Department at Virginia Polytechnic Institute and State University, Blacksburg, VA. He received his B.S. degree in inorganic nonmetallic materials science and engineering from Wuhan University of Science and Technology, Wuhan, China, in 2001, and his Ph.D. degree from Shanghai Institute of Ceramics, Chinese Academy of Science, Shanghai, China, in 2010. From March 2007 through June 2009, he worked as a Research Assistant in Department of Applied Physics at the Hong Kong Polytechnic University (Poly U), and from June 2008 through September 2009, he worked as a Research Assistant in the Department of Electrical Engineering. His current research interests include magnetoelectric composites, magnetic sensors, piezoelectric materials/actuators, and other functional devices.

Peter Finkel is Research Physicists at the Multifunction Materials Branch at the Naval Research Laboratory. Peter received a $\mathrm{PhD}$ in Materials Science/Low Temperature Physics from Drexel University, a MS in Physics from Queens College at The City University of New York. His research areas include experimental solid state physics, magnetism, and materials science, with focus on magnetic and ferroelectric materials, sensors, transduction and ultrasonics. His work concentrates on investigation of single crystal piezoelectric materials used in acoustic devices and novel magnetoelectric sensors. Prior to joining NRL Dr. Finkel was a Materials Scientist and R\&D Scientist in the Devices, Sensors and Materials R\&D Branch at the Naval Undersea Warfare Center (NUWC) in Newport, Rhode Island and Physicist at the RCA/GE/Thomson R\&D Center, Lancaster, PA. He has authored more than 35 refereed publications and has delivered many invited lectures and seminars.

Jiefang Li received her Ph.D. degree in solid-state science from The Pennsylvania State University. She is currently a research professor of Materials Science and Engineering at Virginia Tech. Her research interests include ferroelectric, piezoelectric, dielectric, and magnetoelectric materials. She has been instrumental in the development and study of magnetoelectric laminate composites. Jiefang has published more than 100 peer-reviewed journal articles.

Dwight Viehland is currently in the Department of Materials Science and Engineering at Virginia Tech. He received B.S. and M.S. degrees from the University of Missouri-Rolla, and a Ph.D. from The Pennsylvania state University. Dwight is an experimental solid-state scientist in the structure and properties of condensed matter and thin layers. His research focuses on sensor materials including magnetoelectricity, piezoelectricity, and magnetostriction. Since joining Virginia Tech, the Viehland laboratory began a new area of research that involved the development of novel materials and composites with large magneto-electric exchanges. 